\title{
COLABORACIÓN ESPECIAI
}

\section{POPPER Y EL PROBLEMA DE LA INDUCCIÓN EN EPIDEMIOLOGÍA}

\author{
José R. Banegas, Fernando Rodríguez Artalejo y Juan del Rey Calero \\ Departamento de Medicina Preventiva y Salud Pública. Facultad de Medicina. Universidad Autónoma de Madrid.
}

\section{RESUMEN}

En este artículo discutimos algunas aportaciones del filósofo austro-británico Karl R. Popper, uno de los más influyentes pensadores contemporáneos, cuyas teorías epistemológicas y sociopolíticas han llegado también al ámbito de la epidemiología. Nos centramos principalmente en el llamado problema de la inducción.

Sostenemos, siguiendo a Popper que el método científico no usa un razonamiento inductivo, sino hipotético-deductivo. Aunque el paso desde los datos que evalúan una hipótesis a una conclusión sobre ésta va de lo particular a lo general, e.d., en dirección iuductiva, no existe la inducción como razonamiento o inferencia. Es decir, no existe un método que permita inducir o verificar las hipótesis o teorías (no es posible explorar todas las situaciones posibles para ver si la teoria se mantiene), ni siquiera hacerlas muy probables. Además, los científicos buscan teorías altamente informativas, no altamente probables.

Lo que se hace realmente es proponer una hipótesis como solución tentativa de un problema, confrontar la predicción deducida de la hipótesis con la experiencia, y evaluar si la hipótesis queda rechazada o no por los hechos. Al no poder verificarse las teorías sólo podemos aceptarlas si resisten el intento de rechazarlas. Por tanto, la contrastación consiste en la crítica o intento serio de falsación, es decir, la eliminación de error dentro de una teoría, para rechazarla si es falsa. El objetivo es, pues, la búsqueda de teorías verdaderas.

Para ello, el método científico utiliza un conjunto sistemático de reglas metodológicas (no lógicas), es decir, decisiones. Estas reglas o principios metodológicos se resumen en dos; jsea inventivo y crítico!, e.d., proponga hipótesis audaces y sométalas a tests rigurosos de la experiencia. La lógica juega principalmente su papel al permitir deducir de la hipótesis las predicciones que se confrontarán con los hechos o evidencias. Esto es aplicable tanto a la inferencia estadística como a la inferencia causal.

Argumentamos que los criterios de causalidad usados en epidemiología no son sino reglas del método destinadas a lo mismo: tratan de eliminar o reducir el error (azar, sesgos...) al contrastar una hipótesis causal. Por tanto, la llamada inferencia causal, el paso de la evidencia a la teoria causal, no es un proceso lógico inductivo o probabilístico sino decisión basada en la evaluación de una hipótesis causal gracias a reglas metodológicas como los criterios de causalidad.

Pensamos que el interés del debate entre los epidemiólogos poppericuos y los inductivistas no es meramente verbal, pues si somos conscientes de que no operamos inductivamente, que no podemos cstableccr firmemente una hipótesis, ni siquiera afirmarla probabilísticamente, seremos presuntamente más humildes en nuestra actitud y buscaremos más los errores en las teorías que sus fáciles ejemplos confirmadores.

Correspondencia:

José R. Banegas

Arzobispo Morcillo s/n. 28029 Madrid. Tel.: 397.5425.

Fax: 397.5353.

Correo electrónico: joseramon.banegas@uam.es.

\section{ABSTRACT

Popper and the Problem of Induction in
Epidemiology

In this article we are discussing a few of the contributions by the Austro-British philosopher Karl R. Popper, one of our most influential contemporary thinkers, whose epistemological and socio-political theories have also penetrated the sphere of epidemiology. We are focusing mainly on the so-called problem of induction.

We sustain, in line with Popper, that the scientific method does not use inductive reasoning, but rather hypothetical-deductive reasoning. Although the movement from the data evaluating a hypothesis to a conclusion on the latter goes from the specific to the general, that is, in an inductive direction, the induction does not exist as a reasoning process or inference. That is, there is no method that enables us to infer or to verify hypotheses or theories (we cannot explore all of the possible situations to see whether the theory stands up), or even to render them very probable. Besides, scientists look for highly informative theories, not highly probable ones.

What we actually do is to propose a hypothesis as a tentative solution to a problem, to confront the prediction deduced from the hypothesis with actual experience, and evaluate whether the hypothesis is rejected or not by the facts. As theories cannot be verified, we can only accept them if they withstand an attempt to reject them. Consequently, the test of a theory consists of criticism or a serious attempt at falsification, that is, the elimination of error within a theory, in order to reject it if it is false. The objective is, thus, the search for true theories.

For this purpose, the scientific method uses a systematic set of methodological (not logical) rules, that is, decisions. These methodological rules or principles can be summed up in two: ; be inventive and critical!, that is, propose bold hypotheses and subject them to severe tests of experience. Logic plays its role mainly by allowing us to deduce from a hypothesis the predictions to be confronted with the facts or evidence. This is applicable both to statistical inference as well as to causal inference.

We argue that the criteria of causality used in epidemiology are none other than rules of the method designed for the same purpose: they are concerned with eliminating or reducing errors (chance, bias ..) on testing a causal hypothesis. Consequently. the so-called ausal inference, the step from evidence to causal theory, is not a logical inductive or probabilistic process but rather a decision based on the evaluation of a causal hypothesis thanks to methodological rules such as the criteria of causality.

We believe that the interest of the debate between the Popperian and the inductivist epidemiologists is not merely a matter of words, as, if we are aware that we do not operate inductively, that we cannot establish firmly hypotheses, not even affirm them probabilistically, we will presumably adopt a humbler attitude and look more for the errors in our theories than for their facile examples of confirmation. 
Aunque no podamos justificar racionalmente nuestras teorias, y ni siquiera probar que son probables, podemos criticarlas de forma racional y objetiva, buscando y eliminando errores al servicio de la verdad, distinguiendo así entre teorías mejores y peores

Karl Raimund Popper

\section{Lo cierto será siempre penúltimo y lo último siempre será incierto \\ Pedro Laín Entralgo}

En este artículo vamos a recordar la excursión que algunos sanitarios han hecho al popperiano mundo de las conjeturas y refutaciones, el viaje de algunos inquietos epidemiólogos al país de la epistemología - la moderna filosofía de la ciencia- en busca de las bases científicas de su disciplina de estudio y, por qué no?, parafraseando al propio Popper, en busca de un mundo mejor ${ }^{1}$.

Influyente en el pensamiento de ilustres científicos, economistas y políticos ${ }^{2 \cdot 5}$, formados en los seminarios o en las lecturas del Popper de la London School of Economics en los años 50 y 60 , el pensamiento del filósofo trascendió en sus quehaceres. Si bien se hizo esperar algo más, la influencia popperiana en el terreno de la salud pública, y especialmente en el de la epidemiología, se introdujo formalmente a mediados de los años 70. Un artículo de Carol Buck en el International Journal of Epidemiology en $1975^{6}$ avivó el interés y desató la polémica sobre los variados puntos de vista de la filosofía de la inferencia causal en Epidemiología. Y decimos que lo avivó, y no que lo inició, pues el debate era previo aunque no estaba tan preeminentemente centrado en la figura de Popper $^{7,8}$; y esta discusión no es exclusiva de la epidemiología sino de todas las ciencias, al menos las fácticas ${ }^{9}$.

El objetivo de este ensayo es analizar la influencia de Popper en la epidemiología contemporánea, centrándonos principalmente en el llamado problema de la inducción, uno de los problemas fundamentales de la epistemología y que ha sido invocado con frecuencia en el tratamiento de la causalidad en epidemio$\operatorname{logía}^{10}$.

\section{EL PROBLEMA DE LA INDUCCIÓN. INDUCCIÓN Y LÓGICA}

La tensión dialéctica entre los detractores ${ }^{8,9.11-13}$ y partidarios de Popper ${ }^{6.14-18}$ gravita sobre la aceptación o rechazo, respectivamente, de que es posible aprender inductivamente de la experiencia, es decir de la idea intuitiva de que a medida que se van acumulando los datos que confirman una teoría, aumenta la probabilidad de que ésta sea verdadera. Punto que rechaza Popper al arguir que no existe fundamento lógico ni psicológico para la probabilidad inductiva: Sir Karl aboga por la imposibilidad de alcanzar inductivamente conocimiento cierto o incluso probable (en sentido matemático) a partir de los siempre finitos datos de la experiencia; pues siempre podría haber una nueva observación que refutara la proposición y no existe tal cosa como la probabilidad inductiva (en el sentido del cálculo de probabilidades).

En lugar de la inducción, Popper se decantó para la aproximación a la verdad de una hipótesis por el método hipotéticodeductivo $^{19,20}$, sólo la lógica deductiva es el órgano crítico. De una hipótesis a contrastar, en conjunción con las condiciones iniciales del estudio, se deducen lógicamente una o varias predicciones; luego éstas se confrontan con la experiencia de los estudios emprendidos para contrastar rigurosamente la hipótesis. Si las predicciones concuerdan con los resultados del estudio, se decide que quedan verificadas y la hipótesis queda provisionalmente corroborada; pero si la decisión es negativa, la teoría de la que se han deducido queda deductivamente falsada (rechazada), aunque también provisionalmente. En este proceso no aparece la lógica inductiva (razonamiento o inferencia de las observaciones a la hipótesis) por ninguna parte.

Mas, aunque ambas son provisionales, falsación y corroboración no son lógicamente equivalentes, la primera es más fuerte que la segunda. Es decir, hay una asimetría del razo- 
namiento lógico (deductivo): un conjunto finito de observaciones, sí son verdaderas (no sesgadas), puede falsar una hipótesis universal; pero bajo ninguna condición (incluso si observaciones repetidamente verdaderas) podría verificarla (no podemos escudriñar todos los lugares y tiempos para ver si aparece un contraejemplo). $\Lambda$ sí, si una teoría concuerda con los hechos la teoría puede todavía ser falsa (sus predicciones puede ser explicadas por otras hipótesis alternativas), lo más que podemos esperar es su plausibilidad provisional corroboración en términos popperianos-; si, por el contrario, no hay acuerdo, la teoría es necesariamente falsa (salvo que lo falso sean las condiciones iniciales, e.d, las observaciones estén sesgadas). Por ello, las hipótesis científicas, de una manera válida, sólo pueden ser refutadas, falsadas, nunca se confirman; con lo que únicamente quedan provisionalmente preferidas aquellas que resisten repetidamente fuertes intentos de refutación (pruebas empíricas).

Pero si se trata de una hipótesis probabilista (no determinista), desde un punto de vista lógico no es verificable (por ser universal) ni tampoco estrictamente falsable (pues por improbables que sean las desviaciones considerables de los datos respecto a lo predicho por la hipotesis en una muestra grande, una hipótesis no excluye lógicamente dichas desviaciones) ${ }^{9,19-21}$. Pero sí son falsables con arreglo a reglas metodológicas (p.e. las desviaciones grandes o los niveles de significación estadística). Y la asimetría lógica anterior se mantiene.

Que una hipótesis no puede ser deducida con fuerza lógica de los datos a su favor es algo que nadie cuestiona: ningún conjunto finito de datos puede confirmar una hipótesis (una hipótesis universal afirma una relación válida, en principio, en todo espacio y tiempo) $)^{9,19-21,22}$. Pero el aserto popperiano de que las hipótesis científicas no puedan ser justificadas ni siquiera como probables por inducción ha sido contestado, sobre todo en el marco filosófico del bayesianismo ${ }^{11,23}$. También su solución positiva para el apoyo de una hipótesis (no meramente la negativa de ausencia de falsación) por medio de medidas de corroboración que no son probabilidades, como alternativas a la probabilidad inductiva, ha sido desafiada ${ }^{11,24}$.

\section{El interés del debate}

Muchos científicos siguen creyendo en la inducción porque consideran que la ciencia natural se caracteriza por el método inductivo, es decir por un método que parte de largas series de observaciones y experimentos y se basa en ellos ${ }^{25}$. Creen que sólo el método inductivo puede suministrar un criterio de demarcación entre ciencia y especulación metafísica o seudociencia, y que este método permite establecer o verificar como seguras, o casi seguras o muy probables, las teorías. Mas si lo analizamos cuidadosamente los casos aparentes de inducción no son sino el método de ensayo y error, de conjeturas y refutaciones, es decir el método hipotético-deductivo. En primer lugar, una hipótesis no es generada por los datos sino inventada por los científicos para dar cuenta de las observaciones que forman parte del problema que la hipótesis trata de resolver ${ }^{9,22,2} 5$. Una vez obtenida evidencia pertinente y supongamos que a favor de la hipótesis, no se induce su probabilidad, sino que se cvalúa o juzga la hipótesis a la luz de la evidencia y de argumentos críticos que ayudan a decidir sobre su aceptabilidad. Eso simula la inducción, pues se salta de las observaciones (particulares) a la hipótesis (general), como si dijéramos en dirección inductiva, pero no hay razonamiento o inferencia sino estimación y decisión en dicho paso.

Por lo tanto, el método científico es un conjunto sistemático de reglas metodológicas, e.d. de decisiones dirigidas a asegurar la contrastación empírica de una hipótesis, e.d., la crítica, falsación o eliminación de error dentro de la misma. Las reglas o principios metodológicos se resumen en dos: ¡sea inventivo y crítico!, es decir, proponga hipótesis audaces y sométalas a tests severos de la experiencia. No son reglas lógicas sino metodológicas. El objetivo es la búsqueda de teorías verdaderas ${ }^{19-21}$.

Pero, ¿es el problema de la inducción un tema importante o mera palabrería? ¿Es transcendente este debate en la práctica? ¿Por qué el interés suscitado entre algunos distinguidos 
epidemiólogos desde 1975? Aparte del interés intelectual por conocer mejor las bases epistemológicas de una disciplina científica como la epidemiología, pensamos que el problema de la inducción tiene importancia práctica.

En primer lugar, la inducción predispone a estatuir las teorías lo más posible ${ }^{19-21}$. Pues -dicen los inductivistas-, aunque una teoría bien probada podría tener que ser modificada ante nuevos experimentos, nunca ha ocurrido que una teoría establecida haya tenido que ser derrocada, en todo caso queda restringida a un campo de aplicación más concreto. Sin embargo, la posibilidad de falsar una teoría bien corroborada hace que nadie deba dar por sentado que ha descubierto una ley o teoría verdaderamente universal (p.e. la que relaciona el colesterol sérico con la cardiopatía isquémica), pues no podemos estar seguros de que su validez se extienda más allá de los periodos y sitios en que se observó (p.e. en Framingham -EE.UU.-, Reino Unido y unos pocos lugares más). Considerar que la teoría está corrohorada, que es provisionalmente verosímil, sirve sólo como hipótesis de trabajo, de cara a la acción en otros lugares o circunstancias de aquéllos en los que se testó. A pesar de ello, no añadimos en nuestra formulación de la teoría una condición de que sólo es válida donde y cuando se estudió, porque es un importante postulado del método científico que debemos buscar leyes con un campo de validez ilimitado. Este postulado exige que todo cambio, o modificación de efecto, de una relación sea explicado, porque sin ese postulado bastaría con dar por sentado que las leyes cambian con las diferentes regiones o con el tiempo. Esto vale tanto para las ciencias sociales como para las naturales ${ }^{26}$. En definitiva, como dice Popper ${ }^{19}$, la adoración del ídolo de la certidumbre (verificabilidad) de las teorías (que incluye los grados de certidumbre imperfecta o probabilidad) reprime la audacia de nuestras preguntas y pone en peligro el rigor y la integridad de nuestras contrastaciones.

En segundo lugar, tras este debate puede subyacer también el problema de los límites de la ciencia empírica, no solo de la epidemiología. Si somos conscientes de que no operamos inductivamente, que no podemos esta- blecer firmemente una hipótesis, ni siquiera afirmarla probabilísticamente, seremos presuntamente más humildes en nuestra actitud y buscaremos más los errores en las teorías que sus fáciles ejemplos confirmadores. Ello no impide que afirmemos, provisionalmente, que algunas hipótesis están mejor contrastadas y corroboradas que otras; ni que empleemos la probabilidad (en el sentido matemático) en ese juicio, pero probabilidad de los datos, no de la hipótesis.

Desde luego, los frecuentes debates sobre los límites de la epidemiología ${ }^{27}$, como si no los hubiera, y en grado similar, en otras ciencias empíricas, hace pensar que algunos epidemiólogos buscan una seguridad (inductivismo) en las conclusiones de los estudios epidemiológicos que éstos no parecen proporcionar por la insegura base metodológica subyacente (datos no experimentales, posibilidad de sesgos y múltiples factores de confusión, etc.). Es razonable ser prudente y consciente de los límites de la ciencia epidemiológica, pero no hay razones para serlo mucho más que en otras ciencias empíricas observacionales (p.e. gran parte de la física o la biología molecular, paradigmas de ciencia dura, con gran capacidad demostrativa).

\section{INDUCCIÓN E INFERENCIA ESTADÍSTICA}

El problema de la inducción ha penetrado también, desde hace muchos años, en el terreno de la llamada inferencia estadística o estadística inductiva. Ésta se refiere al contraste de una hipótesis o a la estimación de parámetros en la población a partir de observaciones en muestras extraídas aleatoriamente de la población. En ambas pruebas estadísticas se procede de la evidencia en la muestra a la hipótesis en la población, de lo particular a lo general, esto es en dirección inductiva; pero el razonamiento o inferencia implicados son deductivos.

En los tests de hipótesis, los posibles resultados de un experimento aleatorio y, por tanto, los errores alfa y beta, y los valores p (probabilidad de los datos observados o más extremos, bajo la asunción de verdad de la hipótesis nula), se infieren o calculan por razonamiento lógico 
deductivo a partir del supuesto de verdad de la hipótesis nula y de la hipótesis alternativa. A continuación es solo cuestión de decisión (que es un acto de voluntad), no de inferencia (razonamiento o argumento) deductiva ni inductiva, rechazar o no la hipótesis testada (la hipótesis nula) ante los resultados observados, según una regla metodológica (nivel de significación prefijado), es decir, tras decidir qué constituye un resultado estadísticamente significativo. $\mathrm{O}$ sea, se decide comportarse como si la hipótesis nula fuera falsa y la alternativa verdadera, o vicever$\mathrm{sa}^{18,28,29}$. Aunque Ronald Fisher abogó por un papel inferencial inductivo para los tests de significación ${ }^{30.31}$, hoy día parece más aceptada la visión anteriormente citada ${ }^{23,31}$.

En las pruebas de estimación, los límites de confianza se deducen de ciertas premisas estadísticas (teorema central del límite, distribución normal de la variable aleatoria, o grandes números, etc). A partir de ahí, de nuevo, es cuestión de decisión comportarse como si el valor verdadero del parámetro estuviese situado, en la proporción calculada, dentro de tales límites calculados de las observaciones ${ }^{29,32}$.

Todo ello concuerda con la visión de Popper de que los tests estadísticos son hipotéticodeductivos, que incluyen reglas de decisión, $y$ de que no hay lugar para la inducción ${ }^{19-21}$. No obstante, todo esto se desarrolla en el seno de la llamada estadística frecuentista u objetivista, la que solemos utilizar en nuestra práctica investigadora; otra cuestión, que luego comentamos, es la estadística subjetivista o bayesia$n a$, en la que algunos defienden que hay lugar para el razonamiento inductivo ${ }^{11,23,33,34}$.

Precisamente, que sean decisiones y no conclusiones (deductivas) lo que en último término decide el destino de una hipótesis estadística, habla de que la evaluación de la hipótesis es tentativa, provisional, no concluyente o definitiva, tal como decía Popper ${ }^{19-21}$.

\section{INDUCCIÓN Y PROBABILIDAD}

Aunque empleemos enunciados de probabilidad frecuentista (valores $\mathrm{p}$, intervalos de confianza) para contrastar hipótesis probabilís- ticas, lo que se calcula en la estadística frecuentista es la probabilidad de los datos bajo ciertas hipótesis y no la probabilidad de las hipótesis dados los datos ${ }^{31}$. ¿Cómo apostar, pues, en favor o en contra de una hipótesis?

La preferencia por una determinada hipótesis basada en su probabilidad (en el sentido del cálculo de probabilidades) no es admitida en la epistemología popperiana; no ocurre así en otras escuelas que aceptan basar la credibilidad de una teoría a partir de un juicio de probabilidad inductiva (principalmente bayesiana), es decir de la probabilidad de la hipótesis ante una masa de datos a su favor ${ }^{9,11}$.

Los argumentos de Popper contra el uso de la probabilidad de las hipótesis científicas al evaluar las mismas son, en nuestra opinión, ciertamente dignos de consideración ${ }^{19-21}$. Uno de estos argumentos es que el cálculo de probabilidades es incompatible con la conjetura de que la probabilidad es ampliativa $y$, por tanto, inductiva ${ }^{20,35}$. Esto se puede examinar desde la perspectiva del bayesianismo, o mejor del teorema de Bayes aplicado a la probabilidad de hipótesis como paradigma moderno de inductivismo.

Si se dispone de dos (o más) hipótesis en competencia y hay evidencia favorable a ambas, el teorema de Bayes no permite discriminar entre ambas. Por ejemplo, la evidencia de la relación entre colesterolemia alta y cardiopatía isquémica comprobada en algunos pocos países es compatible tanto con una hipótesis generalizadora o inductiva (la relación se mantiene en todos los lugares y tiempos) como con una hipótesis contraria a la primera, contrainductiva (la relación se mantiene en esos países pero es de signo contrario en otros países o grupos de edad, sexo, etc. en los que todavía no se ha estudiado, o ante otras características biológicas o ambientales no consideradas). El teorema de Bayes no arregla esto: la relación entre las probabilidades de ambas hipótesis a posteriori (es decir, tras considerar la evidencia) es igual a la relación de las probabilidades a priori, dado que las probabilidades de la evidencia ante ambas hipótesis es la misma. Por tanto, la evidencia no apoya a la primera más que a la segunda hipótesis. 
La evidencia no presta apoyo (probabilístico) inductivo a la hipótesis, no apoya nada dentro de la hipótesis salvo la parte del contenido de la hipótesis que incluye la evidencia $^{36}$. No obstante, si una teoría ha sido bien corroborada, es sumamente probable que se parezca a la verdad, es decir que concuerde bien con algunos de los hechos; dicho de otro modo es muy improbable que el éxito sea un mero accidente (azar). Pero no hace probable a la hipótesis, es decir, que sea más probablemente verdadera que falsa, pues esto requeriría que fuera más probable que concordase con todos los hechos, en todos los lugares, tiempos y circunstancias, que lo contrario, que no hay contraejemplo que la contradiga, mas ninguna evidencia finita puede decirnos nunca tal cosa.

$\mathrm{Y}$, sin embargo, de cara a la acción, decidimos que una de las dos hipótesis es preferible de momento: en principio aceptamos la primera (como hipótesis de trabajo, para poder usar la información del colesterol como factor de riesgo coronario) porque la segunda, al ser novedosa, atrevida, tendría que ser explicada y, además, entraría en conflicto con otras teorías bien corroboradas (p.e. los mecanismos de oxidación de las lipoproteínas). Pero hemos de continuar contrastándola.

Popper introdujo la idea de corroboración para mostrar el carácter absurdo de toda teoría probabilística de la preferencia de hipótesis y, por tanto, de toda teoría probabilística de la inducción ${ }^{37}$. Por grado de corroboración entiende un informe conciso que evalúe el estado, en un cierto momento $t$, de la discusión crítica de una teoría respecto al modo en que resuelve sus problemas, su grado de contrastabilidad, el rigor de las contrastaciones a que ha sido sometida y cómo ha salido de ellas. Hay que recordar que ni Popper ni la estadística frecuentista de Von Mises, Neyman, Pearson y otros $^{28,32,38}$ prohiben el uso de la probabilidad en la evaluación de las hipótesis. Las medidas de corroboración o aceptabilidad o preferencia de una hipótesis se basan en la probabilidad, pero probabilidad de los datos bajo la hipótesis, no de la hipótesis bajo los datos (recuérdese el concepto de valor $p$ o de intervalo de confianza). También hay que recordar que no solo las evidencias (bajo las probabilidades) influ- yen en el juicio sobre una hipótesis, sino también los argumentos no estrictamente probabilísticos o cuantitativos (ver criterios de causalidad). Tampoco se prohibe el uso del teorema de Bayes, sino que, como se ha mostrado, su aplicación a la evaluación de las hipótesis no parece fértil.

Otro argumento contra el uso de la probabilidad de las hipótesis al evaluarlas es que los científicos buscan teorías altamente informativas, no altamente probables ${ }^{25}$. Una teoría audaz, cuyo contenido informativo va mucho más allá de toda la evidencia observada (p.e. al postular un nuevo factor de riesgo) es improbable o inesperada a la luz de lo que se sabe en la actualidad, pero fructífera para el avance del conocimiento de resultar cierta.

\section{INDUCCIÓN Y CAUSALIDAD}

Por lo que se refiere a la explicación causal, Popper define el objetivo de la ciencia como la búsqueda de explicaciones (causales) de los fenómenos del cosmos (incluyendo la biología humana), es decir, de las leyes verdaderas que regulan nuestro universo ${ }^{20}$. Es ésta una cuestión de obvio interés en Epidemiología, cuyo propósito más importante es adquirir conocimiento de las causas de las enfermedades que no son prevenibles actualmente ${ }^{39}$.

También en el campo de la causalidad aparece con fuerza el problema de la inducción. Desde Sexto Empírico (200 d.C.) a Hume en 1740 , muchos filósofos han reducido la cuestión de la causalidad a la de regularidad o legalidad; observamos que a un acontecimiento específico suele seguir regularmente otro, pero no observamos un nexo necesario entre ambos. En su búsqueda subjetivista de la idea de necesidad al analizar la causalidad, dice Popper ${ }^{37}$, Hume introdujo el problema de la inducción: si la relación entre A y $B$ fuese necesaria (o incluso solo probable) debería darse (o darse con mayor probabilidad) no solo en los casos pasados, ya observados, sino también en los futuros, no observados; y como no hay fundamento lógico para poder afirmar esto, no es la razón, sino solo la costumbre o hábito lo que nos lleva a esperar que sigan cumpliéndose las 
regularidades; no hay nexo causal, la causalidad es solo regularidad.

La contestación de Popper es ciertamente interesante: no podemos decir que empujar una mesa determinada (suceso A) es la causa de su movimiento (suceso B) si no suponemos que generalmente el efecto sigue a la causa postula$\mathrm{da}$, es decir si no hipotetizamos una ley general. Popper cambia el foco de A y B a su relación; y dice que sí hay vínculo causal (necesario o probable) entre A y $\mathrm{B}$ : éste es la hipótesis general que postula que la relación debe darse (o darse con mayor probabilidad) en toda circunstancia, lugar y tiempo ${ }^{19-21,37}$. Lo postula la hipótesis, pero no se dice que la realidad deba ser así; por eso hay que investigar desafiando la hipótesis, aunque con la esperanza de que se confirme. Así, en términos epidemiológicos, la hipótesis de que el colesterol alto (A) es causa de cardiopatía isquémica (B) no podemos justificarla racionalmente (ni lógica ni empíricamente) pues no podemos observar todos los sujetos, poblaciones o circunstancias en que podrían aparecer contraejemplos. A pesar de ello, formulamos la hipótesis (general) de relación entre A y B; que es tanto más aceptable, satisfactoria, y verosímil, cuanto más y mejor se vea corroborada en sucesivos y variados estudios (consistencia), y cuanto más profunda (plausible) sea la relación (a lo que ayudan los llamados criterios de causalidad, como luego veremos).

Este preámbulo de aclaración conceptual nos permite plantear más nítidamente el debate sobre si la inferencia causal, o los criterios de causalidad, son o no un proceso inductivo. Recordamos que entendemos por inducción la pretensión de ir, con seguridad (o probabilidad), desde lo observado hasta más allá de lo observado, de saltar de la evidencia observada para contrastar una hipótesis a la generalidad o validez universal de ésta (en todos los lugares, tiempos y circunstancias).

El interés de considerar la inducción en su relación con la causalidad en epidemiología se suscita, en parte, porque algunos epidemiólogos se oponen al uso de los criterios de causalidad (como los de $\mathrm{Hill}^{40}$ ), por tacharlos de inductivos $\mathrm{y}$, por tanto, no fructíferos. Pero, a nuestro parecer, el problema se desvanece si consideramos que dichos criterios no son inductivos ( $\mathrm{ni}$ deductivos como pretenden otros). Los criterios causales no son reglas lógicas sino metodológicas, que ayudan en el proceso de decisión sobre la inferencia causal en epidemiología. El papel de la lógica, sea ésta deductiva o inductiva (esta última existe solo para los neobayesianos), se ubica esencialmente en momentos diferentes al de la inferencia de los datos a la hipótesis ${ }^{19,20.23}$. Veámoslo.

Tras disponer de una hipótesis (universal o espaciotemporalmente independiente) ${ }^{19-21,41,42}$ causal como explicación de un problema de salud (p.e., la hipercolesterolemia es causa, probabilística, de la enfermedad coronaria -EC-) hay que deducir de la misma, conjuntamente con el auxilio de condiciones iniciales (las características específicas del estudio diseñado al efecto: p.e., el estudio de Framingham en los años 1940s sobre sujetos blancos, de edad media y nivel social medio), una predicción en un marco geotemporal concreto, que solemos llamar hipótesis operativa (en los sujetos, sitio y tiempo estudiados, la frecuencia de la EC es mayor en aquellos con colesterol alto, o el riesgo relativo es mayor que 1). La predicción se realiza gracias a la lógica genuina, la lógica deductiva: se trata de una inferencia (directa) deductiva. Luego, se confronta la predicción con los datos del estudio. En el proceso de contrastación de dicha predicción con la experiencia procedente del estudio contrastador, ya vimos que la obtención del valor $p$, y del intervalo de confianza se realizan, en el seno de la estadística frecuentista, deductivamente. Además, hay que decidir si la evidencia (los hechos o datos) es aceptada o no como verdadera, asumicndo los consiguientes riesgos de error. Pero este proceso no es lógico sino metodológico, guiado por reglas metodológicas (estadísticas y no estadísticas - p.e., consideración de sesgos-): es una decisión. Una decisión no es un elemento lógico, mas no por eso es arbitrario, se toma sopesando motivos razonadamente con arreglo a reglas; tampoco es, como aseveran algunos autores ${ }^{8,10}$, subjetivo. Hay búsqueda de verdad objetiva, pues la evidencia (y su valoración) es contrastable, discutible intersubjetivamente por cualquiera que conozca las técnicas 
especificadas por los investigadores, o sea, reproducible (aproximadamente) con la ayuda de criterios o reglas metodológicas.

Por último viene el proceso de inferencia (inversa) desde la evidencia a la hipótesis sustantiva (causal). Es este el paso más controvertido filosóficamente, pues para algunos implica confirmación inductiva y para otros fracaso en el intento de refutación deductiva. Parece claro que el paso de la evidencia a la hipótesis ocurre en dirección inductiva, de lo singular a lo general (en términos estadísticos, de la muestra a la población). Para los inductivistas (digamos los neobayesianos) es posible estimar la probabilidad de que una hipótesis (causal) sea verdadera. Esto es lógica inductiva: se espera que los casos no observados seguirán confirmando, probabilísticamente, la hipótesis. Pero en el seno de la estadística frecuentista, y de la inferencia científica que la asume, que son las que empleamos en la práctica, la inferencia no es lógica. No es lógica auténtica, deductiva, pues, de datos a favor finitos no se puede deducir con certeza hipótesis que van más allá de esa experiencia (puede surgir contraejemplos en los casos no observados). La lógica inductiva, ampliativa, no sería posible: aparte de por las razones ya vistas, la probabilidad asume que los datos no observados son similares a los observados (ley de los grandes números o estabilidad de las frecuencias relativas a largo plazo) y, por tanto, no puede probar dicha asunción ${ }^{19-21,38,43,44}$. Además, la lógica inductiva, aunque existiera, no sería necesaria. Bastan las reglas metodológicas para decidir (o inferir) sobre la hipótesis. En realidad se decide sobre la evidencia (estadística y sustantiva) y esto ayuda a estimar o valorar la hipótesis. Por ello, la inferencia, el salto de la evidencia a la hipótesis, es materia de juicio y decisión. Si la hipótesis no quedara rechazada, sino sustentada por evidencia positiva, queda provisionalmente corroborada, aceptada. Y si la hipótesis queda rechazada por evidencia negativa, al ser estadísticas las hipótesis epidemiológicas, no puede quedar falsada lógicamente, concluyentemente, aunque sí metodológicamente en la práctica, con ayuda de reglas. En caso de duda hay que renunciar a tomar un acuerdo y proseguir la contrastación y discusión.
Tanto en la contrastación como en la inferencia causal son útiles, pues, ciertas reglas metodológicas: obtención de un efecto reproducible, evidencia independiente a favor de las condiciones iniciales (e.d., en contra de los sesgos), a favor de la propia hipótesis (exclusión de explicaciones alternativas, plausibilidad), etc. A ello van dirigidos los criterios de causalidad (ver más adelante).

Dos advertencias. Una, que la decisión o juicio sobre una hipótesis (causal o no) sea un proceso metodológico, no significa que la lógica no juegue ningún papel. Cuando hablamos de que una hipótesis está corroborada por la evidencia, sólo la parte observada de la misma (la relación observada en los sujetos estudiados, en el lugar y tiempo de estudio/ estudios) queda deductivamente confirma$\mathrm{da}^{21,36}$, aunque sujeta a revisión por la falibilidad de las observaciones o existencia de mejores hipótesis alternativas. El componente inductivo de la hipótesis (los casos no observados) y, con él, la hipótesis entera, solo quedan corroborados prácticamente, de cara a la acción; pero, teoréticamente, la hipótesis ha de seguir comprobándose, especialmente si surgen nuevas e interesantes oportunidades: nuevos contextos o campos de aplicación, técnicas más precisas de medición, etc. Por tanto, no hay que confundir corroboración lógica, que tiene una parte concluyente desde un punto de vista lógico, con la empírica, que es problemática y siempre inacabada. Cuando una predicción está en contradicción con la evidencia, alguna de las premisas de las que se deriva (la hipótesis o las condiciones iniciales del estudio) es lógicamente (deductivamente) falsa; es cuestión de decisión cuál de ellas se considera falsada: si las condiciones del estudio se aceptan como verdaderas (estudio no sesgado), es la hipótesis la que queda refutada. En todo caso, aun cuando una teoría bien corroborada hasta la fecha quede falsada, nunca se debe abandonar si no hay una mejor teoría de repuesto, que sea más informativa ${ }^{19-21,37}$.

Y segunda, de cara a la acción siempre hay que tomar una decisión (sí/no); p.e. un consejo preventivo se da o no se da. Pero la evaluación de la hipótesis a la luz de la evidencia puede admitir varios valores (p.e. muy, bastante, 
aceptable, poco o nada corroborada), es policotómica o gradual.

\section{Inducción y criterios de causalidad}

Ahora podemos ya afrontar más directamente la pregunta: ¿los criterios de causalidad ${ }^{40}$ son inductivos o deductivos?, subyacente en el debate más general de la inferencia causal ${ }^{10}$.

Parece, tal como lo ven algunos autores ${ }^{10}$, Susser entre ellos ${ }^{12,45}$, que la filosofía popperiana es incompatible, al menos en parte, con los criterios de causalidad, pretendidamente inductivos ${ }^{42}$, propugnados en epidemiología ${ }^{40}$, ya que Popper y sus seguidores ${ }^{14,18-21}$, niegan la posibilidad de la inducción. Pero la llamada metodología popperiana o hipotético-deducti$\mathrm{va}^{19-21,37,46}$, no solo no es inconsistente con los criterios de causalidad (de Hill y otros) ${ }^{40,47}$ sino que subsume los principales criterios como reglas del método científico útiles para progresar en la contrastación y evaluación de una hipótesis causal ${ }^{19-21,37,46}$ (epidemiológica en nuestro caso).

A nuestro entender, la raíz de esta falsa incompatibilidad radica en no haber reconocido (o no haberlo hecho suficientemente), el rol decisional, como reglas metodológicas, de los criterios de causalidad, y su ubicación en el marco metodológico de contrastación científica. Esta solución rompería, adicionalmente, el pseudodebate inductivo o deductivo en la inferencia causal en epidemiología. La mala comprensión también puede venir de la pretensión de que la inferencia causal, como tal inferencia de las observaciones a la hipótesis causal, es inductiva, cuestión que ya discutimos.

\section{La lógica y el método en los criterios de causalidad}

Así pues, algunos autores ${ }^{18,48}$ están en contra del uso de criterios causales, y dicen que la inferencia causal no es sino proponer hipótesis causales y someterlas a severos tests críticos, es decir, la aplicación del método hipotéticodeductivo a la contrastación de hipótesis causales. Para algunos de ellos ${ }^{17}$, no creyentes en la inducción, los criterios causales no deberían usarse iprecisamente por ser inductivos!
Para Greenland ${ }^{34}$ el concepto de inducción de Popper es extraño y, por tanto, está fuera de la auténtica discusión. Pero Popper (y otros autores) no pudo ser más claro acerca de que la probabilidad inductiva, úsese o no en la explicación causal, se refiere a lo que la inducción siempre ha querido significar: pretender saber más de lo que se sabe, siquiera sea de una manera probable, o sea, pretender ir con las evidencias actuales sobre una hipótesis más allá de lo que dicha evidencia dice $e^{9,14,22,35,36,43}$. $\mathrm{Y}$, como ya vimos, la probabilidad lógica (inductiva) dice que si el contenido de la hipótesis va más allá de la evidencia, entonces sólo la parte de la hipótesis que se sigue de la evidencia se hace cierta y el resto sigue tan probable o tan improbable como era sin la evidencia. Nos remitimos a los apartados anteriores que elaboran más esta cuestión, aunque admitimos que sigue abierto a la discusión. No obstante, pensamos que es Greenland, y algunos de los ex-popperianos persuadidos por él, quien tiene el extraño concepto de que la inducción probabilística es un proceso deductivo de actualizar probabilidades a posteriori usando el teorema de Bayes y sus consecuencias ${ }^{34,49}$. Aunque ni el mismísimo Popper ${ }^{19-21,50}$ discute que el teorema de Bayes es deductivo (se deduce de los axiomas de las probabilidades), parece claro en la literatura que su aplicación al cálculo de la probabilidad de una hipótesis (universal) dados los datos no solo va en dirección inductiva sino que de haber razonamiento implicado (cosa que no admiten los popperianos) éste es inductivo ${ }^{11,23,31,33}$. Pensamos que Greenland puede no haber distinguido entre el teorema de Bayes, que es deductivo, y sus aplicaciones.

Otros autores hablan de que los criterios en general tienen una orientación inductiva $o$, lo que es lo mismo, los criterios no se acomodan a la visión de la ciencia de Popper (y otros) $)^{14,19-21}$. Rothman y Greenland ${ }^{42}$ hablan de que, a pesar de las críticas filosóficas a la inferencia inductiva, se usan con frecuencia criterios causales orientados inductivamente. Para Susser y otros ${ }^{10,12,45}$ la consistencia es un ejemplo notorio de criterio inductivo, en tanto la repetición de evidencias a favor de la hipótesis (causal) aumenta la verificación o probabilidad de ésta. Realmente, Susser es ecléctico y hace una aproximación inductivo/deductiva a 
la inferencia causal ${ }^{12,45}$. Finalmente, para $\operatorname{otros}^{15,16,51}$, como refutacionistas, los criterios deberían ser vistos como tests deductivos de hipótesis causales, que ayudan a descartar explicaciones alternativas a la causal.

Para nosotros, los criterios de causalidad, como otras muchas reglas de la ciencia, no fueron concebidos como reglas de inferencia lógica, ni inductiva ni deductiva, sino como reglas metodológicas, reglas de discusión críti$\mathrm{ca}$, es decir criterios que ayudan, no imponen con fuerza lógica (o probable), a decidir si la evidencia apoya o rechaza, o no permite concluir, siempre tentativamente, sobre la hipótesis causal. Como ya vimos, el momento en que actúa la lógica (reglas lógicas o inferenciales) es en el de la deducción de predicciones de una hipótesis o en la falsación deductiva de una hipótesis universal ante una evidencia negativa.

Precisamente que los criterios sean reglas metodológicas, decisionales, explica que puedan tener, como hemos dicho, excepciones. Las decisiones se incluyen en el método crítico de la ciencia, pero siempre como decisiones tentativas y sujetas a la crítica. Nuestras decisiones no tienen por qué ser decisiones últimas $^{52}$. Realmente, si se acepta que tienen esta naturaleza, sobraría tener que justificar que no son necesarios ni suficientes para la causalidad. Por eso ésta es una cuestión de juicio, gradual, aunque dicotómica en la acción. En todo caso, scría preferible evitar el término criterios por su connotación de necesarios y concluyentes (criterio $=$ método definitivo o seguro de decisión) $)^{52}$. Se trata más bien de reglas o normas flexibles, orientadoras, y provisionales.

Y si no son criterios inductivos, no se debería hablar con propiedad de inferencia causal sino de valoración, estimación, cvaluación o juicio causal. El matiz no es meramente lingüístico, pues la segunda concepción reconocería la imposibilidad de saltar de los datos a la causalidad por inferencia lógica (salvo para negarla), segura, ni siquiera probable (en el sentido del cálculo de probabilidades), sino por el más modesto, y no formalizable con números o fórmulas, acto, decisión, de aceptar (o no) la hipótesis causal, tentativa y provisionalmente, de cara a la posible acción.
Es cierto que algunos criterios, como los de consistencia y plausibilidad, a pesar de ser reglas metodológicas, permiten tests deducti$v^{5}{ }^{51}$. Permiten que se deduzcan nuevas consecuencias contrastables de la hipótesis, sean epidemiológicas (en la consistencia) o biológicas (en la plausibilidad). Pero los criterios en sí no son deductivos, son reglas del método, que facilitan la contrastación y evaluación de las teorías; dan nuevas oportunidades para que la hipótesis sucumba o se siga manteniendo, es decir facilitan y se subordinan a la regla metodológica suprema del método científico ${ }^{19-21}$, la contrastación de la hipótesis, y permiten avanzar en su profundización.

Es decir, los principales criterios de causalidad no son sino reglas del método destinadas a lo mismo: tratan de eliminar el error (falta de concordancia con los hechos, sesgos...) al contrastar una hipótesis causal propuesta para resolver un problema. Consideremos algunos de cllos.

Las repeticiones no son confirmaciones inductivas, sino intentos críticos de comprobación de posible error ${ }^{53}$. La repetición o consistencia más fértil será aquella conseguible con diferentes investigadores, en distintos lugares y tiempos de observación (criterio de generalización), con diferentes diseños de estudios (criterio de calidad de la evidencia), en circunstancias variadas (ambientes, conductas, tipos de sujetos observados), al tener en cuenta otros factores de riesgo (criterio de independencia) o al descartar posibles sesgos (criterio de evaluación de la evidencia) $)^{8,10,19-21,37,40}$. Todos ellos son variantes del criterio o regla metodológica de repetición y consistencia. Es en estas situaciones cuando resultados consistentes cuentan como auténtica corroboración de una hipótesis causal, pues entonces la hipótesis se somete a mayor riesgo de ser refutada, ha sufrido exámenes más duros ${ }^{21,22}$. Se reduce la posibilidad de que el azar o los sesgos sean la causa de la asociación ante múltiples estudios y circunstancias.

El criterio causal de plausibilidad biológica tiene interés porque una teoría que especifique el mecanismo de acción es lógicamente más fuerte, más rica (mayor contenido), meto- 
dológicamente más exigente (da más numerosas y precisas oportunidades de contrastación), y suministra una explicación más profunda (detalla un nivel de la realidad distinto, más básico) ${ }^{8,22,37,54}$. La fuerza de la asociación es otro criterio causal ${ }^{40}$. Su regla de interpretación es que cuanto mayor es la fuerza (riesgo relativo) más verosímil es la hipótesis causal. Los confusores rara vez dan cuenta de riesgos muy altos. El criterio de relación dosis-respuesta puede ser considerado como variante del criterio de magnitud ${ }^{55}$. En cuanto al criterio de relación temporal, como dice Susser ${ }^{45}$, es una propiedad esencial de la hipótesis, es parte de su definición.

Muchos criterios de causalidad están interrelacionados. Por ejemplo, la repetición de un estudio en diversas circunstancias es el mejor test de generalizabilidad; y la consistencia, de generalización. Todos apuntan a la regla suprema de la falsabilidad ${ }^{19-21}$. Por eso se puede hablar de una teoría del método ${ }^{19,21,50,54}$.

\section{Recopilando}

En definitiva, la idea principal, imperecedera de Popper se centra en la aproximación paulatina, mas siempre inalcanzable en forma definitiva, a la verdad, que pasa por nuestra liberación del error gracias a la crítica propia y ajena, lo que sólo es posible en una sociedad (científica y general) pluralista, abierta que tolera y critica los erryores de todos. Es la idea básica de la Ilustración, recalcada tantas veces por Popper ${ }^{1,20,5} 6$, de la autoemancipación por el conocimiento, el atreverse a progresar en el conocimiento, y cuyo trasunto epidemiológico es fácil de ver. Esperemos que, tal como se autodefinía, Popper haya sido sólo el penúltimo ilustrado de la razón crítica ${ }^{1}$.

El pensamiento de Popper, con los antecedentes históricos de Sócrates y $\mathrm{Kant}^{56,57}$ también tiene de nuevo su correlato en el campo de los valores y la ética en salud pública. El liberalismo popperiano encajaría bien con las estrategias paretianas y neokantianas de intervención negociada con y sobre el sujeto individualmente considerado, en que la relación beneficio-riesgo y respeto a la dignidad personal es máxima, pero menos con las de acción utilitarista y roseana ${ }^{58}$ sobre amplios grupos de la población, en que los profesionales deciden la acción en la que algunos pueden quedar perjudicados para beneficiar a los más.

Aunque algunas de las tesis centrales de Popper hayan sido ya esbozadas por pretéritos autores $^{59,60}$ (el pensador austrobritánico siempre reclamó independencia, no originalidad de pensamiento, para algunas de sus ideas), es precisamente la didáctica y clara insistencia en que podemos aprender de nuestros errores lo que las hace atractivas y eficaces. Por lo que, como diría Italo Calvino hay que leer, si no releer, a los que como Popper son ya unos clásicos ${ }^{61}$.

\section{BIBLIOGRAFÍA}

1. Popper KR. En busca de un mundo mejor. Barcelona: Paidós; 1994.

2. Medawar PB. Induction and intuition in scientific thought. Philadelphia: American Philosophical Society; 1980.

3. Magee BM. Popper. Londres: Fontana; 1973.

4. Albendea M, Boyer M, Martín Santos L, Muguerza J, Rojo LA, Sánchez de Zavala V, Schwartz P, Barraclough N, Hernán F. Rodríguez J. Simposio de Burgos. Ensayos de filosofía de la ciencia. En torno a la obra de Sir Karl Popper. Madrid: Tecnos; 1970.

5. Schwartz P, Rodríguez Braun $\mathrm{C}$, Méndez Ibisate $F$ (editores). Encuentro con Karl Popper. Madrid: Alianza; 1993.

6. Buck C. Popper's philosophy for epidemiologists. Int J Epidemiol 1975;4:159-168.

7. Platt JR. Strong inference. Science 1964; 146:347-353.

8. Susser M. Causal thinking in the health sciences. Concepts and strategies of epidemiology. Nueva York: Oxford University Press; 1973.

9. Hempel C. Filosofía de la ciencia natural (1966). Madrid: Alianza; 1973.

10. Rothman KJ (Ed.). Causal Inference. Chesnut Hill, MA: E.R.I; 1988.

11. Howson $\mathrm{C}$, Urbach P. Bayesian reasoning in science. Nature 1991;350:371-374.

12. Susser M. Falsification, verification and causal inference in cpidemiology: reconsiderations in 
the light of Sir Karl Popper's philosophy. En: Rothman KJ (editor). Causal Inference. Chesnut Hill, MA: E.R.I; 1988.

13. Greenland S. Induction versus Popper: substance versus semantics. Int $J$ Epidemiol 1998;27:543-548.

14. Miller DW. Critical rationalism. A restatement and defence. Chicago and LaSalle, Illinois: Open Court; 1994.

15. Maclure M. Popperian refutation in epidemiology. Am J Epidemiol 1985:121:343-350.

16. Weed DL. On the logic of causal inference. Am J Epidemiol 1986;123:965-979.

17. Lanes $\mathrm{S}$. The logic of causal inference in Medicine. En: Rothman KJ (editor). Causal Inference. Chesnut Hill, MA: E.R.I; 1988.

18. Poole C. Induction does not exist in epidemiology, either. En: Rothman KJ (editor). Causal Inference. Chesnut Hill, MA: E.R.I; 1988.

19. Popper KR. La lógica de la investigación científica (1959). Madrid: Tecnos; 1985.

20. Popper KR. Realismo y el objetivo de la ciencia. Postscriptum a la lógica de la investigación científica. Vol. 1. Madrid: Tecnos; 1985.

21. Popper KR. Logik der Forschung. 10 Auflage. Tübingen: J.C.B. Mohr; 1994.

22. Bunge M. La investigación científica. Su estrategia y su filosofía. Barcelona: Ariel; 1985.

23. Rivadulla A. Probabilidad e inferencia científica. Barcelona: Anthropos; 1991.

24. Greenland S. Probability versus Popper: an elaboration of the insufficiency of current popperian approaches for epidemiologic analysis. En: Rothman KJ (editor). Causal Inference. Chesnut Hill, MA: E.R.I; 1988.

25. Popper KR. Conjeturas y refutaciones. El desarrollo del conocimiento científico (1972). Barcelona: Paidós; 1989.

26. Popper KR. La miseria del historicismo (1957). Madrid: Alianza; 1992.

27. Taubes G. Epidemiology faces its limits. Science 1995;269:164-169.

28. Neyman J. Frequentist probability and frequentist statistics. Synthese 1977:36:97-131.

29. Rivadulla A. Mathematical statistics and metastatistical analysis. Erkenntnis 1991;34:211-236.
30. Fisher RA. Statistical methods and scientific inference (1956). Oxford: Oxford University Press, 1990.

31. Oakes M. Statistical Inference. Chesnut Hill, MA: E.R.I; 1990.

32. Neyman J. Mathematical statistics and probability. Washington: U.S. Department of Agriculture; 1952.

33. De Finetti B. Probability, induction and statistics. The art of guessing. Londres: John Wiley \& Sons; 1972.

34. Greenland S. Probability logic and probabilistic induction. Epidemiology 1998;9:322-332.

35. Popper KR, Miller D. A proof of the impossibility of inductive probability. Nature 1983;302:687-688.

36. Popper K, Miller DW. Why probabilistic support is not inductive. Phil Trans R Soc Lond A 1987;321:569-591.

37. Popper KR. Conocimiento objetivo. Un enfoque evolucionista (1972). Madrid: Tecnos; 1988.

38. von Mises R. Probability, statistics and truth (1957). Nueva York: Dover; 1981.

39. MacMahon B, Trichopoulos D. Epidemiology. Principles and methods. 2nd edition. Boston: Little, Brown; 1996.

40. Hill $\mathrm{AB}$. The environment and disease: association or causation? Proc Roy Soc Med 1965;58:295-300.

41. Miettinen OS. Theoretical epidemiology. Nueva York: John Wiley \& Sons; 1985.

42. Rothman KJ, Greenland S. Causation and causal inference. En: Rothman KJ, Greenland S. Modern Epidemiology. Second edition. Philadelphia: Lippincott-Raven; 1998:7-28.

43. Ilume D. Tratado de la naturaleza humana (1740). Barcelona: Orbis; 1984.

44. Neyman J. First course in probability and statistics. Nueva York: Henry Holt and Company; 1950.

45. Susser $M$. What is a cause and how do we know one? A grammar for pragmatic epidemiology. Am J Epidemiol 1991;133:635-648.

46. Popper KR. Two new conceptions of causality. A world of propensities. Bristol: Thoemmes; 1990. 
47. Greenland S (editor). Evolution of epidemiologic ideas. Chesnut Hill, MA: E.R.I., 1987.

48. Lanes SF, Poole C. Truth in packaging? The unwrapping of epidemiologic research. J Occup Med 1984;26:571-574.

49. Maclure M. Inventing the AIDS virus hypothesis: an illustration of scientific vs unscientific induction. Epidcmiology 1998;9:467-473.

50. Popper KR. Replies to my critics. En: Schilpp PA (ed.). The philosophy of Karl Popper. Part Three. The philosopher replies. La Salle, Illinois: Open Court; 1976.

51. Weed DL. Causal criteria and popperian refutation. En: Rothman KJ (editor). Causal Inference. Chesnut Hill, MA: E.R.I; 1988.

52. Popper KR. La sociedad abierta y sus enemigos (edición revisada) (1966). Addenda. Barcelona: Paidós; 1994.

53. Popper KR. Búsqueda sin término (1976). Madrid: Tecnos; 1993.
54. Popper KR. The myth of the framework. In defence of science and rationality. Londres: Routledge; 1994.

55. Elwood JM. Causal relationships in Medicine. Nueva York: Oxford Univcrsity Press; 1988.

56. Kant I. Respuesta a la pregunta: ¿Qué es Ilustración? En: Erhard JB, Kant I, Lessing GE, Schiller F, y otros. ¿Qué es Ilustración? Madrid: Tecnos; 1989.

57. Platón. Apología de Sócrates. Barcelona: Orbis; 1984.

58. Rose G. The strategy of preventive medicine. Oxford: Oxford University Press; 1992.

59. Wartofsky MW. Introducción a la filosofía de la ciencia. Madrid: Alianza; 1987.

60. Echeverría J. Filosofía de la ciencia. Madrid: Akal; 1995.

61. Calvino I. Por qué leer a los clásicos. Barcelona: Tusquets; 1993. 